\title{
Quantification Des Stocks De Carbone De Trois Pools Clés De Carbone En Afrique Centrale : Cas De La Forêt Marécageuse De La Likouala (Nord Congo)
}

\author{
Yannick Enock Bocko, Doctorant \\ Université Marien Ngouabi, Faculté des Sciences et Techniques, \\ Laboratoire de Botanique et Ecologie, Brazzaville-Congo \\ Suspense Averti Ifo, Docteur \\ Université Marien Ngouabi, Ecole Normale Supérieure, \\ Département de Sciences et Vie de la terre, Brazzaville-Congo
}

\section{Jean Joël Loumeto, Professeur}

Université Marien Ngouabi, Faculté des Sciences et Techniques, Laboratoire de Botanique et Ecologie, Brazzaville-Congo

\section{doi: 10.19044/esj.2017.v13n5p438 URL:http://dx.doi.org/10.19044/esj.2017.v13n5p438}

\begin{abstract}
The data of carbon stock of central African swamps forests are very few. This study carried out an assessment of the carbon stock of living biomass and coarse woody debris of three biotopes (flooded forest, seasonal flooded forest, and terra firm forest) of Likouala swamp forest (North of Congo). The average of the carbon stock are 190.72 $\pm 98.7 \mathrm{tC} / \mathrm{ha}$, $39.69 \pm 21.24 \mathrm{tC} / \mathrm{ha}$, and $9.45 \pm 6.6 \mathrm{tC} /$ ha respectively for above ground, billow ground, and coarse woody debris. The carbon stock of our swamp forest tends to increase generally from the flooded forest to the terra firm forest. In addition, the average of the coarse woody debris carbon stock increases with the increasing of the above ground carbon stock.
\end{abstract}

Keywords: Central Africa, Likouala, swamp forest, biotopes, carbon stock

\section{Résumé}

Les données portant sur l'estimation des stocks de carbone des forêts marécageuses d'Afrique centrale sont très peu nombreuses. Cette étude a portée sur l'estimation des stocks de carbone des arbres vivants et celui du bois mort dans les trois biotopes caractéristiques (la forêt inondée, la forêt périodiquement inondée et la forêt de terre ferme) de la Forêt marécageuse de la Likouala (Nord-Congo). Les moyennes de stocks de carbone sont de $190,72 \pm 98,7 \mathrm{tC} / \mathrm{ha}$ et $39,69 \pm 21,24 \mathrm{tC} / \mathrm{ha}$ respectivement pour la biomasse (aérienne et souterraine) des arbres vivants et de 9,45 $\pm 6,6 \mathrm{tC} / \mathrm{ha}$ pour le bois 
mort. Les stocks de carbone dans cette zone d'étude tendent à augmenter de la forêt inondée à celle de terre ferme. Et la moyenne de stocks de carbone du bois mort s'accroit avec l'augmentation du stock de carbone aérien des arbres vivants.

Mots clés : Afrique Centrale, Likouala, forêt marécageuse, biotopes, stock de carbone

\section{Introduction}

Le rôle des forêts tropicales dans la régulation du climat et la conservation de la biodiversité n’est plus à démontrer (Pascal, 2003). Cependant, il sied de faire ressortir la perte de leur biodiversité et l'augmentation des émissions des Gaz à Effet de Serre (GES) liées à leurs déforestations et dégradation des forêts dues aux actions anthropiques en zone tropicale (Molto, 2012). La déforestation et la dégradation des forêts contribuent aproximativement de 12 à $20 \%$ aux émissions globales des gaz à effet de serre dans l'atmosphère (Pearson et al., 2005 ; van der Warf et al., 2009). La Convention Cadre des Nations unies sur le Changement Climatique encourage les pays en voie de développement à la REDD+ (FAO, 2015). Cela, exige une quantification des stocks et flux de carbone entre les différents pools forestiers. Mais l'insufisance des données portant sur les stocks de carbone des différents pools forestiers, ainsi que le manque de suivi de leurs variations retardent l'installation du mécanisme REDD+ (FAO, 2015). Des efforts importants ont été faits tant au niveau global que régional pour améliorer les bases de données sur les stocks et les flux de $\mathrm{CO}_{2}$, ceci en intégrant à la fois des méthodes indirectes (télédetection) que directes par des mésures in situ. Malgré ces efforts faits ces dernières années (Lewis et al. 2009 ; Laporte et al., 2010 ; Djuikouo et al., 2010 ; Lewis et al., 2013 ; Slik et al., 2013 ; Ekoungoulou et al. 20015), ces données restent encore faiblement representées par rapport à la grande variabilité des strates forestieres noté par Letouzey (1982), dans le bassin du Congo. Pearson et al. (2005) mentionnent que la biomasse (aérienne et souterraine) et le bois mort font partie des pools clés du carbone forestier responsable des émissions de $\mathrm{CO}_{2}$ atmosphérique provenant de la déforestation et de la dégradation forestière. En revanche, le bois mort demeure jusqu'à l'heure actuelle l'un des pools clés de carbone forestier le moins étudié au monde (woldendorp et al., 2002 ; Ifo, 2010 ; Merganicova, 2010 ; Pfeifer et al., 2015). Pourtant, son stock de carbone oscille entre 1 et 50 Tc/ha (Rice et al., 2004 ; Eaton, 2005) et représente environ 4 à $10 \%$ du stock de carbone total d'un écosystème forestier (Vogt, 1991).

Les études écologiques portant sur l'estimation des stocks de carbone des arbres vivants et du bois mort ont été plus menées en Amérique, en Asie 
et en Europe (Harmon et al., 1986 ; Vogt, 1991 ; Siipola et al., 1998 ; Eaton, 2005 ; Wendy et al., 2006; Baker et al., 2007; Lü et al., 2010 ; Merganicova et Merganic, 2010 ; Yang et al., 2010 ; Ngo et al., 2013). Très peu d'études sont disponibles pour l'Afrique, et ont été plus menées en Afrique centrale : Bartholomew et al. (1953) en République Démocratique du Congo, Djomo et al. (2011) au Cameroun, Gautam et Pietsch (2012) au Gabon et Ifo et al. (2015) au Congo. Par ailleurs, la plus part des données (existantes) portant sur l'estimation des stocks de carbone des arbres vivants et du bois mort ont été plus recueillies dans les écosystèmes forestiers de terre ferme. Pourtant, De Wasseige et al. (2014) souligne une forte réduction du couvert forestier des forêts marécageuses congolaises.

Au regard de ce qui précède, il paraît nécessaire d’estimer les stocks de carbone des forêts du Bassin du Congo en général et ceux des forêts inondables (forêt marécageuse et mangrove) en particulier. L'objectif général de cette étude est de fournir des données sur les trois biotopes caractéristiques de la forêt marécageuse de la Likouala. De manière spécifique il s'agit d'estimer les stocks de carbones des arbres vivants et celui du bois mort des trois biotopes: la forêt inondée, la forêt périodiquement inondée et la forêt de terre ferme. Notre hypothèse de travail est la suivante: Les stocks de carbone des trois pools diffèrent entre les biotopes, et celui du bois mort augmentent avec l'augmentation du carbone des arbres vivants.

\section{Matériel et Méthodes}

\section{Caractérisation de la zone d'étude}

La région de la Likouala s’étend sur $66044 \mathrm{~km}^{2}$ au Nord-Est du territoire, formant ainsi par sa superficie la plus grande région du Congo, avec 19,3 \% de l'espace national ; elle abrite 40\% des forêts Congolaises (Mokemo et al., 2002).

Le climat de la zone est de type équatorial de transition marqué par quatre saisons : une grande saison sèche (Décembre - Mars), une grande saison des pluies (Août - Novembre), une petite saison sèche (Juillet - Août) et une petite saison des pluies (Avril - Juin) (Rakotondrabe et al., 2011). En considérant les 50 dernières années les températures sont relativement élevées dans la région et la moyenne annuelle est de $25,6^{\circ} \mathrm{C}$. Pour la même période les précipitations moyennes annuelles varient entre 1207 et 2068 mm et l'humidité relative est comprise entre 63 et $97 \%$ (ANAC, 2015). La végétation de la Likouala est principalement représentée par les forêts marécageuses (Poulsen et Clark, 2002 ; Betbeder et al., 2014). Ces dernières se caractérisent par la présence de trois biotopes: forêts inondées, forêts périodiquement inondées (localisées le long des cours d’eau et avant la zone 
forestière inondée) et les forêts (mixte et/ou monodominante) de terre ferme (Betbeder et al., 2014).

Les sols de la zone d'étude sont classifiés comme des gleysols, en raison des inondations et de la saturation de l'eau au cours de l'année. Ils présentent souvent un horizon superficiel noir, organique, tourbeux ou semi - tourbeux (Poulsen et Clark, 2002 ; Anonyme, 2006 ; Nkouika, 2011).

Le relief de la zone d'étude est plat. Son altitude varie entre 300 et 400 m (Mokemo et al., 2002).

Le réseau hydrographique comporte deux cours d'eaux principaux : L’Oubangui et la Likouala aux herbes (Mokemo et al., 2002).

\section{Dispositif expérimental}

Des parcelles imbriquées permanentes et temporaires de forme circulaire, distantes de 100 à $200 \mathrm{~m}$ chacune (figure 1), ont été installées sur cinq (5) sites de notre zone d'étude (figure 2).

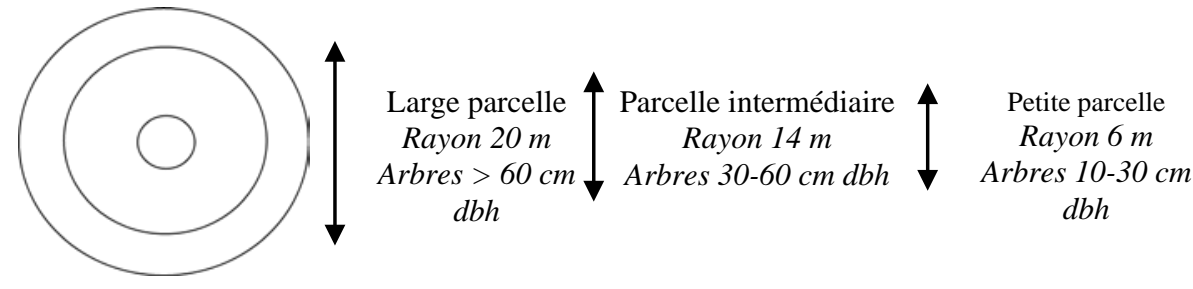

Figure 1 : Illustration d’une parcelle expérimentale

La taille et la forme de ces parcelles représentent un équilibre raisonnable entre l'effort et la précision. L'expérience a montré que les parcelles nids sont d'un coût économique pour les formations forestières (Walker et al., 2011).

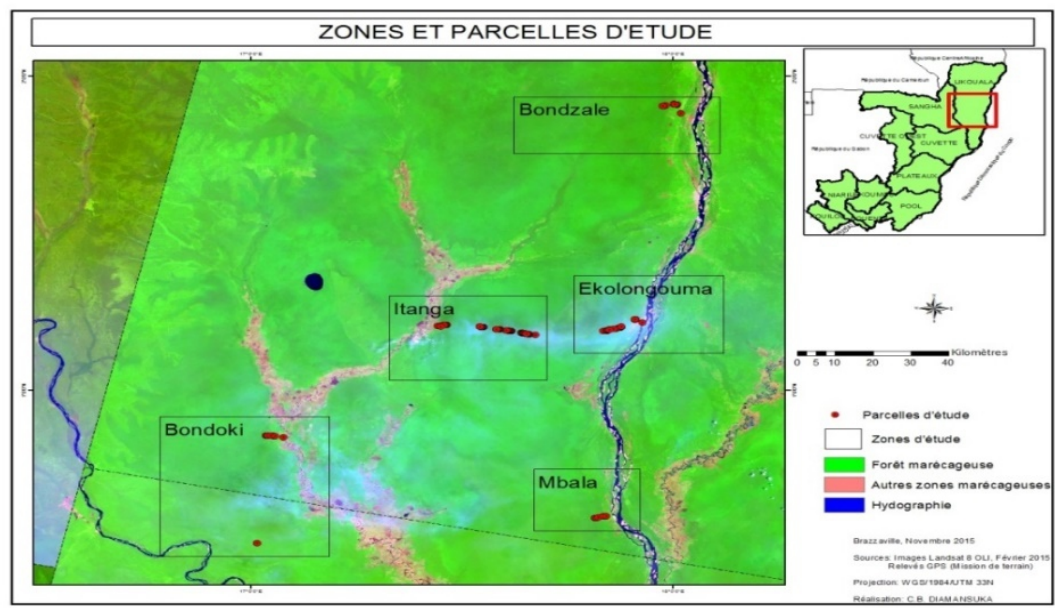

Figure 2: Localisation des sites et parcelles d'étude 
Le Tableau I présente la répartition des parcelles au niveau des différents transects et biotopes de la forêt marécageuse. Au total, 119 parcelles d'échantillonnage ont été installées le long des cinq transects d'étude retenus, à raison de 84, 17 et 18 (respectivement dans la forêt inondée, dans la forêt périodiquement inondée et dans la forêt de terre ferme).

Tableau I : Répartition des parcelles d'étude de carbone par biotope et transect d'étude

\begin{tabular}{|c|c|c|c|c|c|}
\hline \multirow{2}{*}{ Transects } & \multirow{2}{*}{$\begin{array}{c}\text { Longueurs des } \\
\text { transects }\end{array}$} & \multicolumn{3}{|c|}{ Nombre de parcelles par biotope } & \multirow{2}{*}{ Total } \\
\cline { 3 - 5 } & $6 \mathrm{~km}$ & 6 & 4 & 4 & 14 \\
\hline Bondoki & $6 \mathrm{~km}$ & 6 & 4 & 4 & 14 \\
\hline Bondzalé & $9 \mathrm{~km}$ & 6 & 5 & 3 & 14 \\
\hline Ekolongouma & $26 \mathrm{~km}$ & 60 & 4 & 2 & 66 \\
\hline Itanga & $6 \mathrm{~km}$ & 6 & $/$ & 5 & 11 \\
\hline Mbala & & & & & \\
\hline
\end{tabular}

FI : forêt inondée ; FPI : forêt périodiquement inondée ; FTF : forêt de terre ferme

\section{Echantillonnage et estimation du stock de carbone des arbres vivants}

Seuls les arbres de dhp $\geq 10 \mathrm{~cm}$ ont été échantillonnés; et la prise de diamètre des arbres présentant des racines échasses, contreforts et/ou des malformations sur le tronc à $1,30 \mathrm{~m}$ du sol, a été effectuée à $30 \mathrm{~cm}$ au-dessus de ces dernières. Les noms scientifiques et locaux des différents arbres échantillonnés ont été déterminés. Un herbier a été monté sur le terrain afin d'affiner l'identification et la confirmation des noms scientifiques des espèces déterminées et indéterminées. Cette identification et confirmation des noms scientifiques des espèces a eu lieu à Brazzaville au niveau de l'herbier national (IRSEN). La mesure du diamètre des arbres débout ou couchés, mais vivants, s'est faite en prenant en compte les différentes formes des arbres dans la forêt et toutes les mesures de sécurités ont été prises.

La quantification de la biomasse s'est faite en utilisant des équations allométriques génériques. L'équation allométrique de Chave et al. (2014) a été utilisée pour l'estimation de la biomasse aérienne. L'expression mathématique de cette équation allométrique est la suivante :

$$
\begin{aligned}
& \text { AGBest }=\exp [-1,803-0,976 E+0,976 \ln (\rho) \\
& +2,673 \ln (D)-0,0299[\ln (D) 2]] \\
& \text { Où, } E=(0,178 \times T S-0,938 x C W D-6,61 \times P S) \times 10^{-3}
\end{aligned}
$$

Avec $A G B_{\text {est }}$ : estimate aboveground biomass (biomasse aérienne estimée) en $\mathrm{Kg}$;

$\rho:$ la densité spécifique du bois, et $E:$ l'indice climatique liée à l'allométrie auteur diamètre des arbres.

Les densités spécifiques de bois des différentes espèces échantillonnées ont été recueillies de la base des données global wood density database (Zane et al., 2009). La densité moyenne du bois d'Afrique 
$(0,65)$ a été utilisée pour les espèces à densité spécifique inconnue (Lewis et al., 2013). L'extrapolation des valeurs des placettes à l'hectare s'est faite grâce à un facteur d'expansion qui indique la surface représentée par chaque placette. Cette standardisation était nécessaire pour pouvoir aisément interpréter les résultats et aussi faire des comparaisons avec d'autres études.

\section{Facteur d'expansion}

$$
\left.=\frac{10000 \mathrm{~m}^{2}}{\text { Surface de la placette }\left(\mathrm{m}^{2}\right)}, \text { (Walker } \text { et al. }, 2011\right)
$$

La quantification de la biomasse souterraine s'est faite par usage des équations allométriques de Mokany et al. (2006). Les expressions mathématiques de ces équations allométriques sont les suivantes :

$$
Y=0.235 * A G B \text { si } A G B>62.5 t M s / h a(1)
$$

Où $Y:$ la biomasse souterraine en tonnes de matière sèche par hectare (tMs/ha) et AGB : biomasse aérienne estimée.

L'estimation des stocks de carbone de la biomasse aérienne et souterraine s'est faite de la manière qui suit :

Stock de carbone = Biomasse $(M S) * 0,5$ (Brown et al., 2002)

\section{Echantillonnage du bois mort sur pied}

Le bois mort sur pied a été échantillonné comme les arbres vivants de l'écosystème forestier dans 32 parcelles circulaires. Le dhp est mesuré en faisant usage des méthodes relatives aux arbres vivants et la hauteur a été mesurée à l'aide du clinomètre. Le calcul du volume du bois mort sur pied s’est fait grâce à la formule utilisée par Mund (2004) ci-après :

$$
V=\pi * h * f *\left(\frac{\mathrm{dhp}}{2}\right)^{2}
$$

$\mathrm{V}$ : Volume du bois mort sur pied $\left(\mathrm{m}^{3}\right)$; dhp : diamètre à hauteur de poitrine d'un homme (m); h : hauteur du bois mort sur pied (m);

$\mathrm{f}=$ facteur forme $(0.627)$.

\section{Echantillonnage du bois mort couchés au sol}

Le bois mort couché au sol a été mesuré en utilisant la méthode d'intersection des lignes présentée par Harmon et Sexton (1996). Elle a consisté à tracer deux lignes de $50 \mathrm{~m}$ chacune dans chaque direction cardinale (Nord-Sud et Est-Ouest) formant un angle droit au centre d'une parcelle d'étude de biomasse de $20 \mathrm{~m}$ de rayon. Le diamètre de chaque morceau de bois mort, croisant la ligne d'échantillonnage était systématiquement mesuré. Et un morceau de bois mort était mesuré si et seulement si : (1) plus de 50\% du bois mort était au-dessus du sol et (2) la 
ligne d'échantillonnage traversait au moins 50\% du diamètre du morceau de bois mort tombé (Walker et al., 2011). Les différentes parcelles ont été installées au niveau de trois biotopes caractéristiques de la forêt marécageuse de la Likouala : 11 dans la forêt inondée (FI), 9 dans la forêt périodiquement inondée (FPI) et 12 dans la forêt de terre ferme (FTF).

L’équation ci-après a permis de calculer le volume de bois mort accumulé au sol $\varnothing \geq 2.5 \mathrm{~cm}$ :

$\boldsymbol{v}=\frac{\pi^{2}\left(\sum \boldsymbol{d i}^{2}\right)}{\mathbf{8 L}}$ (Waren et Olsen, 1964)

$\mathrm{V}$ : volume de débris ligneux $\left(\mathrm{m}^{3} \cdot \mathrm{ha}^{-1}\right)$; Di : diamètre de chaque débris

ligneux échantillonné $(\mathrm{m})$; L : longueur du transect $(\mathrm{m})=100 \mathrm{~m}$ dans le

cas de notre étude.

La conversion des résultats obtenus du volume en masse s'est faite en fixant la valeur de la densité du bois à 0.47 KgMS.m-3 (Ifo et al., 2010).

- Calcul du stock de carbone du bois mort

Le bois mort (BM) est un pool de carbone. Le passage de la masse sèche au stock de carbone se fait par la relation suivante :

Stock de carbone dans $\mathrm{BM}=$ Quantité de matière sèche (MS) x 0,5 (Woldendorp et al., 2004).

\section{Analyse des données}

L'analyse de différence de stocks de carbone s'est faite d'une part entre les biotopes et d'autre part entre les sites d'étude, grâce au Test de Kruskal-Wallis. Le Test de Wilcoxon-Math-Whitney a été utilisé uniquement lorsque la différence de stock de carbone a été signalée significative après usage du Test de Kruskal-Wallis.

\section{Résultats}

\section{Carbone aérien et souterrain des arbres vivants}

Les moyennes de stocks de carbone aérien et souterrain des arbres vivants de la zone d'étude sont respectivement de l'ordre de 190,72+98,77 tC/ha et 39,69 $\pm 21,24$ tC/ha (Tableau II).

Tableau II : Moyennes de stocks de carbone des arbres vivants des différents biotopes étudiés

\begin{tabular}{|c|c|c|c|c|c|c|c|c|}
\hline \multirow{2}{*}{$\begin{array}{c}\text { Sites } \\
\text { (transects) }\end{array}$} & \multicolumn{2}{|c|}{ Forêt inondée } & \multicolumn{2}{|c|}{$\begin{array}{l}\text { Forêt périodiquement } \\
\text { inondée }\end{array}$} & \multicolumn{2}{|c|}{ Forêt de terre ferme } & \multicolumn{2}{|c|}{ Zone d’étude } \\
\hline & CA (t/ha) & CS (t/ha) & CA (t/ha) & CS (t/ha) & CA (t/ha) & CS (t/ha) & CA (t/ha) & CS (t/ha) \\
\hline Bondoki & $206,2 \pm 46,4$ & $42,3 \pm 9,5$ & $301,9 \pm 62,9$ & $62,9 \pm 13,4$ & $338,3 \pm 102,2$ & $73,8 \pm 2$ & $271,3 \pm 88,2$ & $57,2 \pm 20,1$ \\
\hline Bondzalé & $232,4 \pm 45,8$ & $48 \pm 10,1$ & $269,7 \pm 51,4$ & $55,3 \pm 10,5$ & $334,9 \pm 83,5$ & 19 & $272,3 \pm 70,5$ & $56,9 \pm 16$ \\
\hline Ekolongoum & $210 \pm 34,8$ & $43,1 \pm 7,1$ & $315,1 \pm 73,6$ & $67,7 \pm 16,3$ & 352,1 & & $277,9 \pm 86,8$ & 19,1 \\
\hline & $117,2 \pm 51,6$ & $24,1 \pm 10,6$ & $276,7 \pm 72,2$ & $57,8 \pm 15,4$ & $197,7 \pm 66,7$ & $5 \pm 12,3$ & $129,3 \pm 65,9$ & $26,7 \pm 13,8$ \\
\hline Mbala & $247,7 \pm 56,1$ & $50,8 \pm 11,5$ & 1 & 1 & $234,1 \pm 72,5$ & $49,1 \pm 17,2$ & $241,5 \pm 61,1$ & $49,9 \pm 13,6$ \\
\hline Moyenne & $147,7 \pm 69,7$ & $30,4 \pm 14,4$ & $292,3 \pm 62,8$ & $61,3 \pm 13,8$ & $295,3 \pm 96,3$ & $62,9 \pm 21,7$ & $190,7 \pm 98,8$ & $39,7 \pm 21,2$ \\
\hline
\end{tabular}


Le stock de carbone des arbres vivants de notre zone d'étude est fortement lié à la biomasse aérienne (82,71\%) en comparaison à celui de la biomasse souterraine $(17,29 \%)$. Les moyennes de stocks de carbone aérien et souterrain des arbres vivants dans la forêt inondée $(147,7 \pm 69,7$ tC/ha et 30,4 $\pm 14,4$ tC/ha, respectivement) sont inférieures à celles des arbres des forêts périodiquement inondée (292,3 $\pm 62,8 \mathrm{tC} / \mathrm{ha}$ et $61,3 \pm 13,8 \mathrm{tC} / \mathrm{ha})$ et de

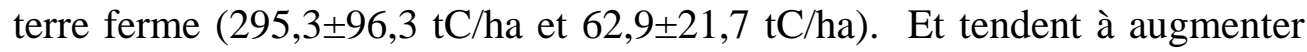
de la forêt inondée à la forêt de terre ferme (Tableau II).

La moyenne du stock de carbone souterrain des arbres vivants dans la forêt inondée est la moitié de celle des forêts périodiquement inondée et de terre ferme. Le test de Wilcoxon- Mann-Whitney a pu révéler d’une part, des différences très significatives entre les stocks de carbone des FI - FPI (pvalue $=0,0001$ ) et entre les FI - FTF (p-value $=0,0001)$, et d'autre part une différence non significative entre stocks de carbone de la FPI et de la FTF (pvalue $=0,7322$ ).

Le Tableau III présente les résultats portant sur l'influence de la distribution diamétrique dans l'estimation des stocks de carbone au niveau des différents biotopes étudiés.

Tableau III: contribution des classes de dhp (cm) des arbres vivants au stockage de carbone

\begin{tabular}{|c|c|c|c|c|}
\hline \multirow{2}{*}{ Biotopes } & \multicolumn{4}{|c|}{ Classes de diamètre } \\
\cline { 2 - 5 } & \multicolumn{2}{|c|}{ Effectifs (\%) } & \multicolumn{2}{c|}{ Stocks de carbone (\%) } \\
\cline { 2 - 5 } & Dhp $<70 \mathrm{~cm}$ & $\mathrm{dhp} \geq 70 \mathrm{~cm}$ & Dhp $<70 \mathrm{~cm}$ & $\mathrm{dhp} \geq 70 \mathrm{~cm}$ \\
\hline Forêt inondée & 95,27 & 4,73 & 87,82 & 12,18 \\
\hline Forêt périodiquement inondée & 82,59 & 17,41 & 61,31 & 38,69 \\
\hline Forêt de terre ferme & 72,08 & 27,92 & 43,15 & 56,85 \\
\hline Moyenne & $\mathbf{8 3 , 3 1}$ & $\mathbf{1 6 , 6 9}$ & $\mathbf{6 4 , 0 9}$ & $\mathbf{3 5 , 9 1}$ \\
\hline
\end{tabular}

Les arbres de dhp $\geq 70 \mathrm{~cm}$ représentent environ $17 \%$ des arbres échantillonnés seulement, et expliquent 35,91\% du stock de carbone aérien de notre zone d'étude. Le pourcentage des individus de $\mathrm{dhp} \geq 70 \mathrm{~cm}$ et de leur stock de carbone, diffèrent d'un biotope à un autre et augmente fortement de la forêt inondée à la forêt de terre ferme (Tableau III).

\section{Carbone du bois mort}

Le Tableau IV présente les résultats portant sur l'estimation de stocks de carbone des différents biotopes étudiés.

Tableau IV : Stocks de carbone du bois mort par biotope étudié

\begin{tabular}{|c|c|c|c|}
\hline Biotopes & BMS (t/ha) & BMP (t/ha) & BMT (t/ha) \\
\hline Forêt inondée & $8,8 \pm 6,7$ & $0,006 \pm 0,004$ & $8,81 \pm 6,2$ \\
\hline Forêt périodiquement inondée & $7,8 \pm 3,7$ & $0,014 \pm 0,009$ & $7,81 \pm 5,5$ \\
\hline Forêt de terre ferme & $11,04 \pm 10,9$ & $0,016 \pm 0,01$ & $11,1 \pm 7,8$ \\
\hline Moyenne & $\mathbf{9 , 4 4} \pm \mathbf{7 , 9}$ & $\mathbf{0 , 0 1} \pm \mathbf{0 , 0 0 8}$ & $\mathbf{9 , 4 5} \pm \mathbf{6 , 6}$ \\
\hline
\end{tabular}

BMS : bois mort couché au sol ; BMP : bois mort sur pied ; BMT : bois mort total 
Les stocks de carbone du bois mort de la zone d'étude varient de

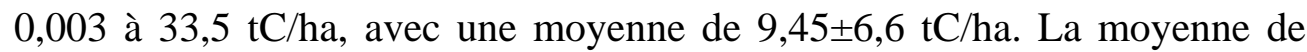

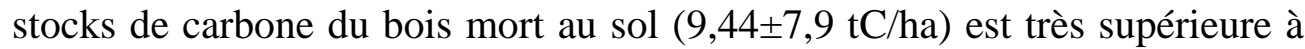

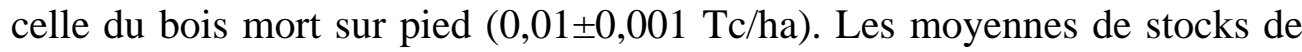
carbone du bois mort de la forêt marécageuse de la Likouala tendent à augmenter de la forêt inondée $(8,8 \pm 6,7 \mathrm{tC} / \mathrm{ha})$ à la forêt de terre ferme $(11,04 \pm 10,9$ tC/ha).

Les moyennes de stocks de carbone du bois mort varient aussi d'un site à un autre (Tableau $\mathrm{V}$ ) ; et présentent une moyenne moins élevée à

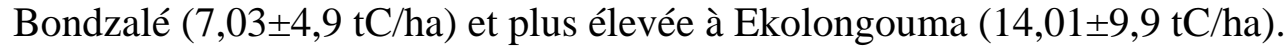

Tableau V : Moyennes des stocks de carbone du bois mort par site (transect) étudié

\begin{tabular}{|c|c|c|c|}
\hline Sites d'étude & BMS (t/ha) & BMP (t/ha) & BMT (t/ha) \\
\hline Bondzalé & $7,02 \pm 6$ & $0,010 \pm 0,009$ & $7,03 \pm 4,9$ \\
\hline Ekolongouma & $14,00 \pm 8,5$ & $0,008 \pm 0,004$ & $14,01 \pm 9,9$ \\
\hline Itanga & $8,7 \pm 6,5$ & $0,016 \pm 0,012$ & $8,72 \pm 6,1$ \\
\hline Mbala & $13,50 \pm 13$ & $0,010 \pm 0,006$ & $13,51 \pm 9,5$ \\
\hline Moyenne & $\mathbf{9 , 4 4} \pm \mathbf{7 , 9}$ & $\mathbf{0 , 0 1 1} \pm \mathbf{0 , 0 0 9}$ & $\mathbf{9 , 4 5} \pm \mathbf{6 , 6}$ \\
\hline
\end{tabular}

L’analyse statistique faite avec le test de Kruskal-Wallis ne révèle pas de différence significative de stocks de carbone de bois mort entre les biotopes d'une part ( $\mathrm{df}=2$, $\mathrm{p}$-value $=0.771$ ) et entre les sites d'étude d'autre part $(\mathrm{df}=3$, $\mathrm{p}$-value $=0.816)$.

\section{Discussion}

\section{Carbone aérien et souterrain des arbres vivants}

Les moyennes de stocks de carbone aérien, obtenus en zone tropicale sont très contrastées. Elles diffèrent suivant les biotopes (Mugnier et al., 2009 ; Lewis et al., 2013 ; Slik et al., 2013). Nos résultats viennent soutenir ces affirmations.

Les moyennes de stocks de carbone trouvées au niveau de la forêt périodiquement inondée et celle de la forêt de terre ferme sont supérieures à d'autres moyennes estimées et obtenues en Afrique centrale (Djuikouo et al., 2010 ; Makana et al., 2011) et au Brésil (Alves et al., 2010). La moyenne de stocks de carbone de la forêt marécageuse de la Likouala est légèrement supérieure à celle trouvées par d'autres Auteurs en Afrique centrale (Mugnier et al., 2009 ; Lewis et al., 2013) et en Asie (Tang et al., 2012 ; Ngo et al., 2013). Plusieurs facteurs pourraient expliquer la variabilité spatiale des stocks de carbone notée au niveau des différents biotopes étudiés. Clark et Clark (2000) et Gourlet-Fleury et al. (2011), soulignent l'influence du type de sol dans la variabilité spatiale de stocks de biomasse, et donc de leur carbone en zone tropicale. En fait, le type de sol contrôle la composition spécifique (Laurence et al., 1999) et explique sa différence entre les biotopes 
par une sélection naturelle liée à l'adaptation des espèces (Jaffré et Veillon, 1990). Marshall et al. (2012) et Shirima et al. (2015) soulignent aussi que les variables structurelles expliquent une plus grande partie de la variation spatiale de la biomasse dans les forêts tropicales africaines. En fait, le stock de carbone aérien tend à augmenter avec l'augmentation de certains paramettres de structure comme la surface terrière (Gourlet-Fleury et al., 2011 ; Rahaingoson et al., 2013). On peut alors noter que les différences les plus significatives entre les stocks de carbone des différents biotopes pourraient résider dans l'importance de leurs surfaces terrières (Jaffré et Veillon, 1990), et de leurs dhp moyens. Ainsi, il est d'une grande nécessité de lier l'estimation de la biomasse aérienne et le stock de carbone à l'étude structurale d'une forêt. Il est aussi admis que la méthodologie d'échantillonnage et le type d'équation allométrique utilisé expliquent les variations de stocks de carbone obtenus en zone tropicale (Clark et Clark, 2002 ; Molto, 2012 ; Komiyama et al., 2008 ; Chave et al., 2004 ; Chave et al., 2008). En effet, Nos parcelles d'étude ne permettent pas un échantillonnage exhaustif comme c'est le cas des parcelles carrées non imbriquées utilisées par d'autres auteurs (Lewis et al., 2009 ; Lewis et al., 2013 ; Slik et al., 2013 ; Makana et al., 2011 ; Ngo et al., 2013 ; ). Et l'équation allométrique généralement utilisée par ces derniers est celle de Chave et al. (2005), tandis que nous avons utilisé lors de notre étude celle de Chave et al. (2014). Cette dernière prend en compte les données d'origine africaine et l'influence du climat de la zone d'étude.

L'étude de la structure diamétrique révèle que les arbres $\mathrm{dhp} \geq 70 \mathrm{~cm}$ de notre zone d'étude contribuent à 35,9\% au stockage de carbone. Nos valeurs avoisinent celles trouvées par de nombreux Auteurs en zone tropicale (Lutz et al., 2012 ; Baishia et al., 2009 ; Djuikouo et al., 2010) et sont inférieures à celles trouvées par Slik et al. (2013) en zone pantropicale. Ces valeurs de notre zone d'étude montrent que la contribution à un stockage de carbone de plus de 30\% par les arbres de dhp $\geq 70 \mathrm{~cm}$ (Brown et Lugo, 1989 ; Clark et Clark, 2000) n'est possible que pour les forêts périodiquement inondée et de terre ferme. Les différences notées dans la contribution au stockage de carbone par les arbres de dhp $\geq 70 \mathrm{~cm}$ s'expliquent par les diversités intra-interspécifique et écosystémique élevée en zone tropicale.

\section{Carbone du bois mort}

Les moyennes de stocks de carbone obtenues lors de la présente étude diffèrent entre les trois biotopes étudiées et tendent à augmenter de la forêt inondée à la forêt de terre ferme. Et on peut aussi noter comme Derrière et al. (2012), que le stock de bois mort, et donc de son carbone, s'accroit avec l'augmentation du stock de carbone aérien des arbres vivants. 
La moyenne de stocks de carbone du bois mort de la zone étudiée est de 9,45 $\pm 6,6$ tC/ha et représente environ $4 \%$ du stock total de carbone. Cela permet d'affirmer comme Vogt (1991), que le stock de carbone du bois mort est d'environ 4 à 10\% du stock total de carbone d'un écosystème forestier.

Les moyennes de stocks de carbone du bois mort sont très contrastées en zone tropicale et sont supérieures à celles trouvées par Vogt (1991) et Gough et al. (2007) en zone forestière tempérée (1,1 tC/ha, respectivement). La moyenne estimée de stock de carbone est supérieure à celles trouvées par certains Auteurs en zone tropicale (Harmon et al., 1986; Siipola et al., 1998 ; Djomo et al., 2011 ; Ifo, 2010 ; Ngo et al., 2013). Elle est égale à celle trouvée par Lü et al. (2010) en Chine et avoisine celles estimées par d'autres auteurs (Fraver et al., 2002 ; Baker et al., 2007 ; Dirocco et al., 2012). Mais elle est inférieure à celles trouvées par de nombreux autres Auteurs (Commings et al., 2002 ; Eaton, 2005 ; Krauss et al. 2005 ; Gautam et Pietsch, 2012 ; Carlson, 2013) et très inférieure à la moyenne du stock de carbone du bois mort estimée par Rice et al. (2004) au Brésil. Ce qui permet de mentionner comme de nombreux Auteurs, que le stock de carbone du bois mort varie entre les types forestiers et entre les sites d'étude (Commings et al., 2002 ; Pearson et al., 2005 ; Eaton, 2005 ; Brandeis et Woodall, 2008 ; Ngo et al., 2013) ; et qu'il n'est pas à négliger lors de l'estimation globale du carbone forestier.

Outres les facteurs cités ci-dessus, d'autres faits pourraient expliquer les différences de stocks de carbone de bois mort notées. Baker et al. (2007) soulignent que les stocks de carbone du bois mort varient suivant les sites et méthodes d'étude. En fait, l'ensemble de nos sites d'étude se localisent dans une vaste forêt marécageuse non interrompue, homogène et généralement inondée (Gilbert, 1984) avec une altitude d'environs $324 \mathrm{~m}$. Mais l'ensemble des sites d'étude ayant servi à l'estimation des stocks de carbone de bois mort en zone tropicale se localisent dans des écosystèmes forestiers de terre ferme (Eaton, 2005 ; Ifo, 2010 ; Djomo et al., 2011 ; Gautam et Pietsch, 2012 ; Carlson, 2013). Les conditions requises pour l'échantillonnage du bois mort au sol peuvent aussi justifier le faible stock de carbone obtenu lors de cette étude en comparaison avec les valeurs trouvées dans la littérature. En effet, la méthode d'échantillonnage nécessite que la longueur de la ligne soit définie sur au moins 100m (Harmon et Sexton, 1996), et un morceau de bois mort ne doit être mesuré que si et seulement si : (1) plus de 50\% du bois mort est au-dessus du sol et (2) la ligne d'échantillonnage traverse au moins $50 \%$ du diamètre du morceau de bois mort tombé (Walker et al., 2011). Ainsi, tout bois mort n'ayant pas permis la prise en compte de ces conditions de mesure n'a pas été échantillonnée. Lors de cette étude nous avons utilisé une ligne de transect de $100 \mathrm{~m}$ seulement par parcelle d'étude, ce qui n'a pas été le cas dans d'autres études en zone tropicale (Eaton, 2005 ; Baker et al., 
2007 ; Ifo, 2010 ; Carlson, 2013). Baker et al. (2007), Ifo (2010) et Carlson (2013) ont utilisé des lignes de transect de $400 \mathrm{~m}, 160 \mathrm{~m}$ et $800 \mathrm{~m}$ par parcelle d'étude, respectivement au sud de Pérou, au Congo et au Gabon. Leurs résultats permettent de souligner que les stocks de carbone de bois mort augmentent donc aussi avec la longueur de la ligne de transect d'étude.

\section{Conclusion}

Les moyennes des stocks de carbone des arbres vivants et du bois mort varient spatialement entre les trois biotopes caractéristiques de la forêt marécageuse de la Likouala ; et augmentent de la forêt inondée à la forêt de terre ferme. Les paramètres structuraux et les méthodes d'échantillonnage expliquent au mieux les différences de stocks de carbone notées entre les trois biotopes étudiés et celles trouvées dans la littérature. La moyenne de stock de carbone du bois mort de la forêt marécageuse de la Likouala représente environs $4 \%$ du stock de carbone total et augmente avec l'augmentation du carbone aérien des arbres vivants.

\section{References:}

1. Alves L.F., Vieira S.A., Scaranello M.A., Camargo P.B., Santos F.A.M., Joly C.A. and Martinelli L.A., 2010. Forest structure and live aboveground biomass variation along an elevational gradient of tropical Atlantic moist forest (Brazil). Forest Ecology and Management, 260, 679-691.

2. ANAC, 2015. Données Météorologique de la région de la Likouala Données météorologiques du district d’Impfondo pour les 50 dernières années. Base de données annuelles de l'agence nationale de l'aviation civile (ANAC), Ministère de transport et de l'aviation civile, Brazzaville, Congo.

3. Angelsen A., Brockhaus M., Sunderlin W.D. et Verchot L.V., 2013. Analyse de la REDD + : Les enjeux et les choix. CIFOR, Bogor, Indonésie, 520p.

4. Baker T.R., Coronado E.N.H., Phillips O. L., Martin, J., Van der Heijden G.M.F., Garcia M., Espejo J.S., 2007.Low stocks of coarse woody debris in a southwest Amazonian forest. Oecologia, 152: 495504.

5. Bartholomew W., Meyer J. and Laudelout H., 1953. Mineral nutrient immobilization under forest and grass fallow in the Yangambi region (Belgian Congo). Pub. Inst. Etude Agron. Congo Beige.

6. Betbeder J., Gond V., Frappart F., Baghdadi N.N., Briant G. and Bartholomé E., 2014. Mapping of Central Africa Forested Wetlands Using Remote Sensing. IEEE Journal of Selected Topics in Applied Earth Observations and Remote Sensing7, 531 (2014). 
7. Brandeis T. J. and Woodall C. W., 2008. Assessment of Forest Fuel Loadings in Puerto Rico and the US Virgin Islands. Ambio Vol. 37, No. 7-8, 6p: 557 - 562.

8. Brown S., 2002. "Measuring carbon in forests: current status and future challenges”, Environmental pollution, 116 (3): 363-372.

9. Brown S., Gillespie A. and Lugo A. E., 1989. Biomass estimation methods for tropical forest with applications to forest inventory data. Forest Science, Vol. 35, pp. 881-902.

10. Carlson B., 2013. Gabon’s Overlooked Carbon. A tropical forest of coarse woody debris. Master: Environmental Management degree, Duke University. 38p.

11. Chave J., Réjou-Méchain M., Búrquez A., Chidumayo E., Colgan M.S., Delitti W.B.C., Duque A., Eid T., Fearnside P.M., Goodman R.C., Henry M., Martínez-Yrízar A., Mugasha W. a, Muller-Landau H.C., Mencuccini M., Nelson B.W., Ngomanda A., Nogueira E.M., Ortiz-Malavassi E., Pélissier R., Ploton P., Ryan C.M., Saldarriaga J.G., Vieilledent G., 2014. Improved allometric models to estimate the aboveground biomass of tropical trees. Glob. Chang. Biol., 20, 3177-3190. doi:10.1111/gcb.12629

12. Chave J., Andalo C., Brown S., Cairns M.A., Chambers J.Q., Eamus D., Fölster H., Fromard F., Higuchi N., Kira T., Lescure J. -P., Nelson B.W., Ogawa H., Puig H.,Riera B. et Yamakura, T., 2005. Tree allometry and improved estimation of carbon stocks and balance in tropical forests. Oecologia, 145: 87-99.

13. Chave J., Condit R., Aguilar S., Hernandez A., Lao S. and Perez R., 2004. Error propagation and scaling for tropical forest biomass estimates. The royal society, 12p.

14. Chave J., Olivier J., Bongers F., Châtelet P., Forget P.M., der Meer P.V., Norden N., Riéra B. and Charles-Dominique P., 2008. Aboveground biomass and productivity in a rain forest of Eastern South America. Journal of Tropical Ecology, 24:355-366.

15. Clark D.B. and Clark D.A., 2000. Landscape-scale variation in forest structure and biomass in a tropical rain forest. Forest Ecology and Management, $137: 185$ - 198.

16. Cummings L. D., Kauffman B. J., Perrry A. D. and Hughes F. R., 2002. Aboveground biomass and structure of rainforests in the southwestern Brazilian Amazon. Forest Ecology and Management, 163 (2002) 293 - 307.

17. Daniel V., 2000. Si la la forêt s’écoule... Quels fondements pour la gestion forestière Française après les tempêtes ? Rapport scientifique WWF, Paris, 26p. 
18. Daniel V., Jean A. and Jacques B., 2002. Deadwood, a vital attribute of old-growth forest biodiversity, a gap in managed forest. Rapport scientifique WWF, Paris, 34 p.

19. De Wasseige C., Flynn J., Louppe D., Hiol Hiol F., Mayaux Ph., 2014. Les forêts du bassin du Congo - Etat des forêts 2013. Weyrich, Belgique, 328p.

20. Derrière N., Wurpillot S. et Vidal C., 2012. Bois mort en forêt. Le numéro 29 (de juin 2012) de l'institut nationale de l'information géographique et forestière (L’IF), 8p. www.ign.fr

21. Dirocco L. T., 2012. A Thorough Quantification of Tropical Forest Carbon Stocks in Malaysia. Carbon Stocks of Tropical Forests, 18p.

22. Djomo A. N., Knohl A. and Gravenhorst G., 2011. Estimations of total ecosystem carbon pools distribution and carbon biomass current annual increment of a moist tropical forest. Forest Ecology and Management, 261:1448-1459.

23. Djuikouo M.N.K., Doucet J.L., Nguembou C.K., Lewis S.L. and Sonke B., 2010. Diversity and aboveground biomass in three tropical forest types in the Dja Biosphere Reserve, Cameroon. Afr. J. Ecol., 48, 1053-1063.

24. Dupuy B., Loumeto J. J., Amsallem l., Gérard C., Nasi R., Maitre H. -F., Réolon F., Fargeot C., Jori F., 1999. Gestion des écosystèmes forestiers denses d'Afrique tropicale humide. 2. Congo. Les Bibliographies du CIRAD ; Ouvrage, Montpelier, Cirad-forêt, 1-32 : $146 p$.

25. Eaton, M.J., 2005.Woody Debris and the Carbon Budget of Secondary Forests in the Southern Yucatán Peninsular Region.Thesis.University of Virginia, $84 \mathrm{p}$

26. Ekoungoulou R., Niu S., Loumeto J. J., Ifo A. S., Bocko Y. E., Mikieleko K. E. F., Guiekisse M. D. E., Senou H. and Liu X., 215. "Evaluating the Carbon Stock in Above-and Below-Ground Biomass in a Moist Central African Forest." Applied Ecology and Environmental Sciences, vol. 3, n 2 : 51-59. doi: 10.12691/aees-3-24.

27. FAO, 2011. Rapport de la neuvième édition biennale de la situation des forêts du monde : Année internationale des forêts. Rome, Italie, $193 p$.

28. FAO, 2015. Considérations techniques relatives à l'établissement de niveaux d'émissions de référence pour les forêts et/ou niveaux de référence pour les forêts dans le contexte de la REDD+ au titre de la CCNUCC. Programme ONU-REDD. CH-1219 Châtelaine, Genève, Suisse 44p. www.fao.org/publications (www.un-redd.org).unredd.org) 
29. Fraver S., Wagner R.G. and Day M., 2002. Dynamics of coarse woody debris following gap harvesting in the Acadian forest of central Maine, U.S.A. Canadian Journal of Forest Research. 32: 2094-2105.

30. Gautam S. and Pietsch A. S., 2012. Carbon pools of an intact forest in Gabon. African Journal of Ecology 50:414-427.

31. Gilbert G., 1984. La masse forestière Congolaise : Son implantation et ses divers faciès. Bois et Forêts des Tropiques (FRA) ; Vol. 204, 319 : $17 p$.

32. Girardin J. A. C., Malhi Y., Aragao L. E. O. C., Mamani M., Huasco H. W., Durand L., Feeley K. J., Rapp J., Silva-Espejo J. E., Silman M ., Salinas N. and Whittaker R . J., 2010. Net primary productivity allocation and cycling of carbon along a tropical forest elevational transect in the Peruvian Andes. Global Change Biology (2010), doi: 10.1111/j.1365-2486.2010.02235.x.

33. Gough C.M., Vogel C.S., Kazanski C., Nagel L.N., Flower C.E, Curtis P.S., 2007. Coarse woody debris and the carbon balance of a north temperate forest. Forest Ecology and Management, 244: 60-67.

34. Gourlet-Fleury S., Rossi V., Rejou-Mechain M., Freycon V., Fayolle A., Saint-Andre L., Cornu G., Gérard J., Sarrailh J.M., Flores O., Baya F., Billand A., Fauvet N., Gally M., Henry M., Hubert D., Pasquier A. and Picard N., 2011. Environmental filtering of densewooded species controls above-ground biomass stored in African moist forests. Journal of Ecology, 99, 981-990.

35. Harmon M.E. and Sexton J., 1996. Guidelines for Measurements of Woody Detritus in Forest Ecosystems (US LTER Publication No. 20) US LTER Network Office, University of Washington, Seattle, WA, USA. 73 pp.

36. Harmon M.E., Franklin J.F., Swanson F. J., Sollin P., Gregory S. V., Lattin J. D. Anderson N.H., Cline S.P., Aumen N.G., Sedell J. R., Lienkaaemper G. W., Cromack K. and Cummins K. W., 1986. Ecology of coarse woody debris in temperate ecosystems. Ecol. Res.15 : 133-302.

37. Ifo A. S., 2010. Apport de carbone au sol et stock dans deux types forestiers (forêt galerie et forêt secondaire) des plateaux tékés. Thèse, Université-Marien-NGouabi, Brazzaville, 194 p.

38. Ifo, A. S., Koubouana, F., Jourdain, C., \& Nganga, D. (2015). Stock and Flow of Carbon in Plant Woody Debris in Two Different Types of Natural Forests in Bateke Plateau, Central Africa. Open Journal of Forestry, 5, 38-47. http://dx.doi.org/10.4236/ojf.2015.51005

39. Jaffré T. et Veillon J.M., 1990. Etude floristique et structurale de deux forêts denses humides sur roches ultrabasiques en Nouvelle 
Calédonie. Rapports scientifiques et techniques, sciences de la vie botanique $\mathrm{n}^{\circ} 3,45 \mathrm{p}$.

40. Karsenty A., 2015. Émissions, déforestation et spéculation. Alternatives Internationales Hors série $\mathrm{n}^{\circ} 017$ novembre.

41. Keller M., Palace M., Asner G. P., Pereira R. and Silva J. N. M., 2004. Coarse woody debris in undisturbed and logged forests in the eastern Brazilian Amazon. Global Change Biology 10:784-795.

42. Komiyama, A., Ong, J.E., Poungparn, S., 2008. Allometry, biomass, and productivity of mangrove forests: A review. Aquat. Bot. 89, 128137.

43. Kraus, K.W., Doyle, T.W., Twilley, R.R., Smith, J.T., Whelan, K.R.T., Sullivan, J., 2005. Woody Debris in the mangrove forests of south Florida. Biotropica. 37: 9-15.

44. Laurance W.F., Fearnside P.M., Laurance S.G., Delamonica P., Lovejoy T.E., Rankin-de Merona J.M., Chambers J.Q. and Gascon C., 1999. Relationship between soils and Amazon forest biomass: a landscape-scale study. Forest Ecology and Management 118 (1999) $127-138$.

45. Lescure J.-P., Puig H., Riéra B., Leclerc D., Beekman A. et Bénéteau A., 1983. La phytomasse épigée d'une forêt dense en Guyane française. Acta Oecol./Oecol. Gen. 4, 237-251.

46. Letouzey R., 1982. Manuel de Botanique Forestière d'Afrique Tropicale. Livre: Botanique Générale, Tome 1. Centre Technique Forestier Tropical, 2ème édition, nº $9661,(131$ - 140) 195p.

47. Lewis S.L., Sonke B., Sunderland T., Begne S.K., Lopez-Gonzalez G., van der Heijden G.M.F., Phillips O.L., Affum-Baffoe K., Baker T.R., Banin L., Bastin J.F.B., Beeckman H., Boeckx P., Bogaert J., De Cannière C., Chezeaux E., Clark C.J., Collins M., Djagbletey G., Djuikouo M.N.K., Droissart V., Doucet J.L., Ewango C.E.N, Fauset S., Feldpausch T.R., Foli E.G., Gillet J.F., Hamilton A.C., Harris D.J., Hart T.B., Haulleville T., Hladik A., Hufkens K., Huygens D., Jeanmart P., Jeffery K.J., Kearsley E., Leal M.E., Lloyd J., Lovett J.C., Makana J.R., Malhi Y., Marshall A.R., Ojo L., Peh K.S.-H., Pickavance G., Poulsen J.R., Reitsma J.M., Sheil D., Simo M., Steppe K., Taedoum H.E., Talbot J., Taplin J.R.D., Taylor D., Thomas S.C., Toirambe B., Verbeeck H., Vleminckx J., White L.J.T., Willcock S., Woell H. and Zemagho L., 2013. Above-ground biomass and structure of 260 African tropical forests. Phil. Trans. $R$. Soc. B. 368 : 20120295. http://dx.doi.org/10.1098/rstb.2012.0295

48. Lü T. X., Yin X. J., Jepsen R. M. and Tang W. J., 2010. Ecosystem carbon storage and partitioning in a tropical seasonal forest in 
Southwestern China. Forest Ecology and Management 260 (2010) 1798-1803.

49. Lutz J.A., Larson A.J., Swanson M.E. and Freund J.A., 2012. Ecological Importance of Large-Diameter Trees in a Temperate Mixed-Conifer Forest. PLoS ONE 7(5): e36131. doi:10.1371/journal.pone.0036131.

50. Makana J.R., Ewango N.C., McMahon S.M., Thomas S.C., Hart T.B. and Condit R., 2011. Demography and biomass change in monodominant and mixed old-growth forest of the Congo. Journal of Tropical Ecology, 27:447-461.

51. Maniatis D., Malhi Y., Saint-André L., Mollicone D., Barbier N., Saatchi S., Henry M., Tellier L., Schwartzenberg M. and White L., 2011. Evaluating the Potential of Commercial Forest Inventory Data to Report on Forest Carbon Stock and Forest Carbon Stock Changes for REDD+ under the UNFCCC. International Journal of Forestry Research, Article ID 134526, 13p. doi:10.1155/2011/134526

52. Merganicova K. and Merganic J., 2010. Coarse woody debris carbon stocks in natural spruce forests of Babia hora. Journal of Forest Science, 56, (9): 397-405.

53. Mokany K., Raison R.J. and Prokushkin A.S., 2006. Critical analysis of root: shoot ratios in terrestrial biomes. Global Change Biology, 12: 84-96.

54. Mokemo G., Moussonissa R. et Mandavo J., 2000. Géographie au $\mathrm{CM}, 1$ volume. INRAP/EDICEF, Collection n²6-Edition n03, 128p.

55. MOLTO Quentin, 2012. Estimation de biomasse en forêt tropicale humide: Propagation des incertitudes dans la modélisation de la distribution spatiale de la biomasse en Guyane française. Thèse : Physiologie et biologie des organismes, populations, interactions ; Université des Antilles et de la Guyane, 184p.

56. Mund M. (2004). Carbon Pools of European beech forests (Fagus sylvatica) under different silvicultural management. Thesis. der Georg-August-Universität Göttingen. 268 p.

57. Ngo K. M., Turner B. L., Muller-Landau H. L., Davies S. J., Larjavaara M., Hassan N. F. b. N. and Lumd S, 2013. Carbon stocks in primary and secondary tropical forests in Singapore. Forest Ecology and Management, 296: 81-89.

58. Nkouika D. N. G., 2011. Monographie départementale de la Likouala; Etude du Secteur Agricole - République du Congo. Rapport de la mission sur l'atelier de restitution et de discussion du projet de monographie agricole départementale de la Likouala ; 95p. 
59. Pascal J. P., 2003. Description et Dynamiques des Milieux Forestiers. Notions sur les structure et dynamique des forêts tropicales humides. Rev. For. Fr. LV - numéro spécial 2003: 118 - 130.

60. Pearson T., Harris N., Shoch D., Brown S., 2005. A sourcebook of methods and procedures for monitoring and reporting anthropogenic greenhouse gas emissions and removals associated with deforestation, gains and losses of carbon stocks in forests remaining forests, and forestation. Global Observation of Forest and Land Cover Dynamics, 25p.

61. Poulsen J. et Clark C., 2002. Etude de faisabilité de la réserve communautaire du Lac - Télé. Rapport, 148p.

62. Rahaingoson F., Rakotoarimanana V., et Edmond R., 2013. Analyse structurale et floristique de la végétation selon les différents types de gestion sur le Plateau Calcaire Mahafaly. Rôle et place des transferts de gestion des ressources naturelles renouvelables dans les politiques forestières actuelles à_Madagascar. <cirad-00933717>. 8p.

63. Rakotondrabe G., Ndiamanana R., Ngele H., Otto F., Bondeko G., Twagirashyaka F., Mobongo R., Dongo S. et Engoumbe G., 2011. Plan d'aménagement et de gestion simplifié de la macrozone d’Impfondo (Lac - Télé et Lac - Tumba) ; 69p.

64. Rice A., Pyle E. and Saleska S., 2004. Carbon balance and vegetation dynamics in an old-growth Amazonian forest. Ecological Applications, 14:S55-S71.

65. Siipola A.L., Siitonen J., Kallio R., 1998.Amount and quality of coarse woody debris in natural and managed coniferous forest near the timberline in Finnish Lapland.Scand. Jour. For. Res.13: 204-214.

66. Slik J.W. F., Paoli G., McGuire K., Amaral I., Barroso J., Bastian M., Lilian Blanc L., Bongers F., Boundja P., Clark C., Collins M., Dauby G., Ding Y., Doucet J.L., Eler E.,

67. Ferreira L., Forshed O., Fredriksson G., Gillet J.F., Harris D., Leal M., Laumonier Y., Malhi Y., Mansor A., Martin E., Miyamoto K., Araujo-Murakami A., Nagamasu H., Nilus R., Nurtjahya E., Oliveira A., Onrizal O., Parada-Gutierrez A., Permana A., Poorter L., Poulsen J., Ramirez-Angulo H., Reitsma J., Rovero F., Rozak A., Sheil D., Silva-Espejo J., Silveira M., Spironelo W., Hans ter Steege, Stevart T., Navarro-Aguilar G.E., Sunderland T., Suzuki E., Tang J., Theilade I., van der Heijden G., Valkenburg J.V., Do T.V., Vilanova E., Vos V., Wich S., Wöll H., Yoneda T., Zang R., Zhang M.G. and Zweifel N., 2013. Large trees drive forest aboveground biomass variation in moist lowland forests across the tropics. Global Ecology and Biogeography, (Global Ecol. Biogeogr.), 11p. 
68. Tang J.W, Yin J.X, Qi J.F, Jepsen M.R \& Lü X.T, 2012. Ecosystem carbon storage of tropical forests over limestone in Xishuangbanna, Sw China. Journal of Tropical Forest Science 24(3): 399-407.

69. Vogt K.A., 1991.Carbon budgets of temperate forest ecosystems. Tree Phys. 9: 69-86.

70. Walker S., Pearson T., Harris N., Sean G., Silvia P., Felipe C. and Sandra B., 2011. Procédures Opérationnelles Standards pour la Mesure du Carbone Terrestre. Document de Winrock international, 76p.

71. Warren W. and Olsen P., 1964. A line intersect technique for assessing logging waste. Forest Science, 10:267-276.

72. Woldendorp G., Keenan R. J., Barry S., Spencer R. D., 2004. Analysis of sampling methods for coarse woody debris. Forest Ecology and Management. 198:133-148.

73. Woldendorp G., Keenan R. J., Ryan M. F., 2002. An Analysis of sampling Methods for Coarse Woody Debris in Australian Forest Ecosystems A Report for the National Greenhouse Strategy, Module 6.6 (Criteria and Indicators of Sustainable Forest Management). 94p.

74. Woodall W. C. and Liknes C. G., 2008. Climatic regions as an indicator of forest coarse and fine woody debris carbon stocks in the United States. Carbon Balance and Management, 8p. http://www.cbmjournal.com/content/3/1/5.

75. Yang F. F., Li L. Y., Zhou Y. G., Wenigmann O. K., Zhang Q. D., Wenigmann M., Liu Z. S. and Zhang M. Q., 2010. Dynamics of coarse woody debris and decomposition rates in an old-growth forest in lower tropical China. Forest Ecology and Management, 259 (2010) 1666-1672.

76. Zanne A.E., Lopez-Gonzalez G., Coomes D.A., Ilic J., Jansen S., Lewis S.L., Miller R.B., Swenson N.G., Wiemann M.C. \& Chave J., 2009. Global wood density database. Dryad digital repository [www document]. URL http://hdl.handle.net/10255/dryad.235 